\title{
A landmark blocks searching for a hidden platform in an environment with a distinctive shape after extended pretraining
}

\author{
Murray R. Horne and John M. Pearce \\ Cardiff University, Cardiff, Wales
}

\begin{abstract}
In the blocking phase of three experiments, rats had to find a submerged platform beneath a spherical landmark in one corner of a triangular pool. Prior to this treatment, they were required to find the platform relative to either a sphere above it (blocking groups) or a rod attached to it (control groups). The position of the platform changed from trial to trial for the initial training. The sphere did not restrict learning about the geometric cues provided by the triangular arena in the blocking phase when 12 sessions of initial training took place in either the triangular (Experiment 1) or a circular (Experiment 3) pool. Blocking was observed, however, after 24 sessions of initial training in either the triangular (Experiment 2) or the circular (Experiment 3) pool. Thus, blocking of geometric cues by a landmark is possible after extended initial training with the blocking cue.
\end{abstract}

In Stage 1 of a blocking experiment, animals receive one stimulus, A, paired with reinforcement. In Stage 2, they receive A in compound with a novel stimulus, $\mathrm{X}$, followed by reinforcement. The presence of A in Stage 2 has been shown to reduce or block learning about X (Kamin, 1969a, 1969b). This form of cue competition has been found in many species, including fish (Tennant \& Bitterman, 1975), birds (Mackintosh \& Honig, 1970), and mammals (Kamin, 1969a, 1969b). Blocking has also been found with such diverse procedures as taste aversion conditioning (Willner, 1978) and the acquisition of causal judgments (Larkin, Aitken, \& Dickinson, 1998). However, in certain spatial tasks, blocking is absent. The present set of experiments explored one explanation for these failures to find blocking.

An example of a failure to find blocking in a spatial task is provided by Hayward, McGregor, Good, and Pearce (2003). During Stage 1, rats were trained in a rectangular pool to swim to a landmark situated above a submerged platform. In Stage 2, the landmark and platform were always located in one corner of a triangular pool. The presence of the landmark during Stage 2 failed to restrict learning about the shape of the pool for finding the platform. This effect has been replicated using a triangular pool in Stage 1 and a rectangular pool in Stage 2 (Hayward, Good, $\&$ Pearce, 2004). A failure to find blocking has also been replicated in an appetitive experiment in which distinctive featural panels located at each corner of a rectangular enclosure failed to block learning about the shape of the environment (Wall, Botly, Black, \& Shettleworth, 2004). Thus, it appears that the presence of a landmark near a goal does not block learning about the position of the goal with reference to the shape of the environment - in other words, learning based on geometric cues. For a review, see Cheng and Newcombe (2005).

This is not to say that blocking in the spatial domain does not occur; on the contrary, blocking among spatial cues has been reported on a number of occasions. For example, Roberts and Pearce (1999) trained rats during Stage 1 in a swimming pool where they were required to swim to a beacon attached to a submerged platform, with a curtain surrounding the pool to restrict visual access to distal landmarks. During Stage 2, the curtain was drawn open, and the beacon and platform remained in the same place with respect to distal landmarks. A control group received only Stage 2 training. A subsequent test with the beacon and platform removed from the pool revealed that the rats who received prior training with the beacon spent significantly less time in the vicinity of where the platform had been previously located than did the control group. This outcome suggests that prior establishment of a beacon as a signal for reinforcement blocked learning about distal cues when beacon and cues were presented together during the second stage of training. Moreover, blocking has been observed among distal landmarks (Rodrigo, Chamizo, McLaren, \& Mackintosh, 1997) and between intramaze and extramaze cues (Chamizo, Sterio, \& Mackintosh, 1985). Furthermore, there has been one instance of blocking of geometric cues by colored walls in a swimming pool (Pearce, Graham, Good, Jones, \& McGregor, 2006). All of these results suggest that spatial cues compete for associative strength in the same way that stimuli compete for associative strength in Pavlovian conditioning.

An important question that arises from these mixed results is whether learning about geometric cues is gov-

M. R. Horne, hornemr@cardiff.ac.uk; J. M. Pearce, pearcejm@cardiff.ac.uk 
erned by principles different from those that apply to other spatial cues. Apart from the one instance of blocking of geometric cues (Pearce et al., 2006), all other studies have failed to show blocking. We shall return later to discuss the implications of the findings by Pearce et al. (2006), but for the time being we will focus on one possible explanation for the failures to find blocking of geometric cues. Miller and Shettleworth (2007) recently put forward a formal model of how learning takes place in environments with a distinctive shape, a model that assumes that changes in the associative properties of geometric cues are governed by the same principles that apply to all other cues. That is, when two or more cues, including geometric cues, signal where a goal is located, the cues must compete for whatever control they acquire over behavior, in the manner predicted by the Rescorla-Wagner (1972) theory. A clear prediction from this model is that pretraining with a nongeometric cue should block learning about a geometric cue provided by the shape of the environment, if both cues should subsequently signal where a goal can be found. To explain the failures to confirm this prediction, Miller and Shettleworth proposed that the nongeometric cue lacked sufficient associative strength when it was paired with the geometric cue.

According to current theories of learning (e.g., Rescorla \& Wagner, 1972), blocking will be more effective, and hence more likely to be detected, when the associative strength of the blocking cue is high at the outset of Stage 2. There are at least two reasons why the associative strength of the landmark might have been low at the start of Stage 2 in the experiments in which blocking of geometric cues was not observed. First, the change in context from Stage 1 to Stage 2 might have resulted in a generalization decrement in the associative properties of the landmark. In all reported spatial blocking experiments with geometric cues, there has been a change of contexts between Stages 1 and 2. For instance, Pearce, Ward-Robinson, Good, Fussell, and Aydin (2001) conducted Stage 1 in a circular pool and then conducted Stage 2 in a triangular pool. The change in context between Stages 1 and 2 may have disrupted the association between the beacon and the location of the platform formed during Stage 1, and thus decreased the likelihood of detecting blocking. Second, it is possible that subjects received insufficient training with the landmark during Stage 1. The number of Stage 1 training trials in the experiments that failed to reveal blocking varied from 20 (Pearce et al., 2001) to 56 (Wall et al., 2004). Even though there was evidence in these experiments that this training was sufficient to encourage rats to approach the blocking cue, it is possible that the associative properties of the cue were only just sufficient to produce this effect and were still a considerable distance from an asymptotic value that could be achieved if substantially more training had been given.

The present experiments explored both of these possibilities for the failure of a landmark to block geometric cues. By conducting Stages 1 and 2 in the same context, Experiment 1 attempted to minimize the possibility that blocking of geometric cues would fail because of a gen- eralization decrement. Experiments 2 and 3 examined the effects of using extended Stage 1 training, with the aim of increasing the likelihood that the associative strength of the landmark would be high at the outset of Stage 2 and thus would enable effective blocking of the geometric cue.

\section{EXPERIMENT 1}

A blocking group received Stage 1 training in an isosceles triangle shaped pool (see Figure 1) with a submerged escape platform located randomly in one of the two equal-angled corners. A spherical landmark was always located above the platform, no matter its location. During this stage, only the landmark could be used to locate the platform. For the second stage, this group received the platform located in just one of the equal-angled corners beneath the same landmark. Both Stages 1 and 2 occurred in the same context to minimize any generalization decrement as a result of the transition from Stage 1 to Stage 2, and presumably maintain the high associative strength of the spherical landmark. Two control groups were trained in Stage 1 identically to the blocking group, except that a rod-shaped landmark was attached to the platform. In Stage 2, the control groups were trained with the platform in one of the equal-angled corners of the triangle, where geometry was the only reliable indicator of the location of the platform. To equate the three groups as closely as possible in the change they experienced at the transition from Stage 2 to the final test trial, each control group was exposed to one or two spherical landmarks that were unre-

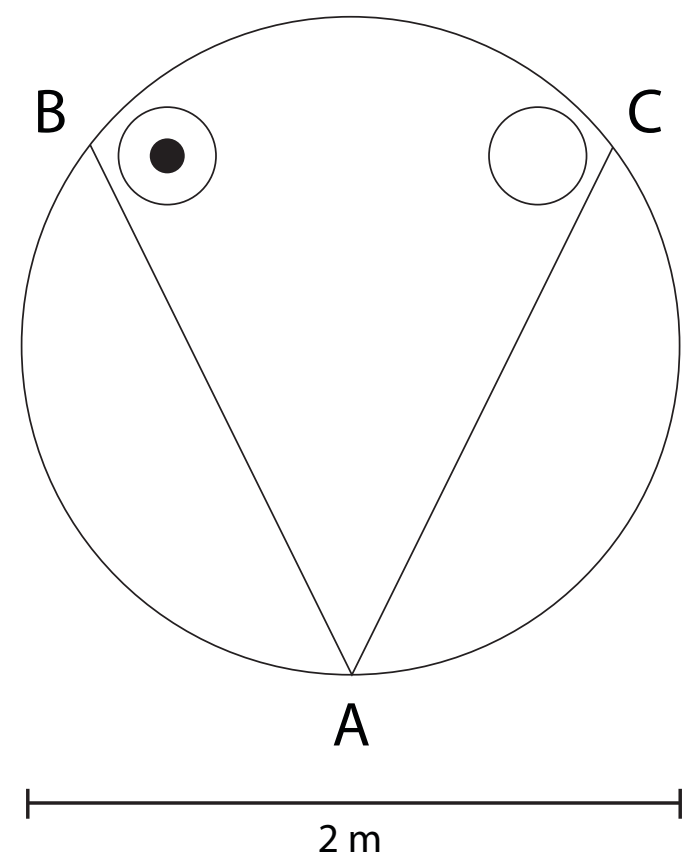

Figure 1. A plan of the apparatus. $A, B$, and $C$ represent the corners of the triangular-shaped swimming pool. The black circle represents the platform location, and the white circles represent search zones, $30 \mathrm{~cm}$ in diameter, in the correct and incorrect locations used for the analysis. 
liable cues for finding the platform. For one control group, a single landmark was located randomly across trials in each of the two equal-angled corners of the pool. A second control group was trained in Stage 2 with two identical landmarks, one over the correct corner that contained the platform and one in the opposite, incorrect, corner. In order for the control groups to find the platform accurately, the geometric cues of the correct corner must be relied upon. Following training, all groups received a 60 -sec test trial in a triangular pool with the platform and landmarks removed.

It must be noted that the typical control for a blocking experiment, in which the control group receives the exact same training as the experimental group during Stage 2, was omitted in this experiment. With the traditional control, the possibility of observing blocking in the blocking group may be diminished because overshadowing by the landmark in the control group might reduce, perhaps considerably, the associative strength of the geometric cue. Both of the control groups we chose here do not pose this problem. For the two control groups to master their tasks, they must learn about the location of the platform with respect to the geometry of the environment. The possibility of observing blocking will then be enhanced, since the associative strength of the target cue will theoretically be at a higher value than if the conventional control condition had been employed. These would be particularly strong control groups if we were to observe a null result.

\section{Method}

Subjects. Twenty-seven male hooded Lister rats (Rattus norvegicus), obtained from Harlan Olac (Bicester, Oxfordshire) and weighing between 250 and $300 \mathrm{~g}$ at the start of the experiment, were used. All rats were previously trained in an appetitive conditioning experiment and had no previous training in a swimming pool. The rats were housed in white plastic cages with secured metal grid lids and maintained on a 12:12-h light:dark cycle with lights on at 0700 . The subjects were housed in pairs and had continuous access to food and water in their home cages.

Apparatus. A white circular pool measuring $2 \mathrm{~m}$ in diameter and $0.6 \mathrm{~m}$ in depth was used. The pool was mounted on a platform $0.6 \mathrm{~m}$ from the floor in the middle of the room $(4 \times 4 \times 2.3 \mathrm{~m})$. The pool was filled with water to a depth of $27 \mathrm{~cm}$ and was maintained at a temperature of $25^{\circ} \mathrm{C}\left( \pm 2^{\circ} \mathrm{C}\right)$. To make the water opaque, $0.5 \mathrm{~L}$ of white opacifier E308 [Rohm and Haas (U.K.) Ltd., Dewsbury, West Yorkshire] was used. The water was changed daily.

A white circular ceiling, measuring $2 \mathrm{~m}$ in diameter, was suspended $1.75 \mathrm{~m}$ above the floor of the pool. In the center of the ceiling was a hole measuring $30 \mathrm{~cm}$ in diameter in which a video camera with a wide-angled lens was situated. The lens of the camera was $25 \mathrm{~cm}$ above the hole and was connected to a video monitor and computer equipment in an adjacent room. During tests, the rats' movements were analyzed using WaterMaze software (Morris \& Spooner, 1990). The pool was illuminated by eight $45-\mathrm{W}$ lights that were located in the circular ceiling above the pool. The lights were $22.5 \mathrm{~cm}$ in diameter and were equidistant from each other in a $1.6-\mathrm{m}$ diameter circle whose center was coincident with the center of the circular ceiling. A platform measuring $10 \mathrm{~cm}$ in diameter and mounted on a column was used during all training trials. The surface of the platform had a series of concentric ridges. For all trials, the base of the column rested on the bottom of the pool and the platform surface was $2 \mathrm{~cm}$ below the surface of the water. Two different types of landmarks were used in the present experiment. One landmark, attached to the platform, consisted of a plastic rod painted with alternating black and white, 1 -cm-wide stripes. A white disk, $3 \mathrm{~cm}$ in diameter and $0.5 \mathrm{~cm}$ thick, was attached to the top of the landmark. This landmark was attached to the platform, $2.5 \mathrm{~cm}$ from its edge. The other landmark was a black sphere, $8 \mathrm{~cm}$ in diameter, attached to the end of a clear plastic rod. The lowest point of the sphere was $33 \mathrm{~cm}$ above the surface of the water on a line that bisected the corner, at a distance of $25 \mathrm{~cm}$ from the corner. The clear plastic rod attached to the sphere was used to hold the sphere in place by being fixed to the top of one of the Perspex boards creating the shape of the pool. A white curtain was drawn around the pool during all training and test trials. The curtain, which was attached to the edge of the circular ceiling, was $1.5 \mathrm{~m}$ high and fell $25 \mathrm{~cm}$ below the edge of the pool.

The training room was also lit by two $1.53-\mathrm{m}$ strip lights connected end to end on each of the east and west walls. These lights ran parallel with the floor and were situated $75 \mathrm{~cm}$ above the floor. There was a door $(1.75 \times 2 \mathrm{~m})$ in the center of the south wall. To create the triangular-shaped pool, two white Perspex boards (1.8 $\mathrm{m}$ long, $59 \mathrm{~cm}$ high, and $2 \mathrm{~mm}$ thick) were placed vertically in the pool and suspended by bars that extended over the edge of the pool.

Procedure. There were two stages of training in the experiment. Stages 1 and 2 consisted of 12 and 14 sessions of training, respectively. Rats completed 1 session of training each day. Each session contained four trials. Rats were brought into a room adjacent to the test room, in groups of 4 or 5 in a light-tight box. They remained in this box between trials. Each rat was carried from the box to the pool and was released facing the center of a wall. The release point varied across trials with the stipulation that each wall was used once in any given session and one release point was used twice. The release point that was used twice varied between sessions such that over 3 sessions, each release point was used four times. During a trial, the rat was required to swim to a submerged platform. Each trial lasted a maximum of $60 \mathrm{sec}$. If the rat did not find the platform within $60 \mathrm{sec}$, the experimenter guided it to the platform. After climbing on the platform the rat remained there for $20 \mathrm{sec}$ before being lifted from the pool, dried, and returned to its holding container. The intertrial interval for each rat was approximately $5 \mathrm{~min}$. Between each trial, the experimenter rotated the arena $90^{\circ}, 180^{\circ}$, or $270^{\circ}$ clockwise. Four possible orientations were used (north, south, east, or west). The orientation of the triangular arena across trials varied randomly with only the stipulation that each orientation was used once for any given session. The center of the platform was situated $25 \mathrm{~cm}$ from the correct corner on a line that bisected the corner.

Rats were randomly assigned to each of three groups in equal numbers ( $n \mathrm{~s}=9$ ): blocking, control-1, and control-2 (the " 1 " and " 2 " refer to the number of landmarks present during Stage 2 training). During Stage 1, all rats in the blocking group received the spherical landmark directly over the platform in corner B for half of the trials, and the platform and spherical landmark were in corner $\mathrm{C}$ for the remaining trials (see Figure 1). The location of the platform and landmark varied randomly, with the requirement that each corner was used twice in a given session. Both control groups received the same training, except that the spherical landmark was replaced with a rod-shaped landmark attached to the platform. After 12 sessions of training, all rats proceeded to Stage 2.

During Stage 2, in the blocking group, 5 of the rats always had the spherical landmark over the platform in corner B, whereas the remaining 4 rats had the spherical landmark and the platform in corner $\mathrm{C}$. For the control-1 and control-2 groups, the platform was located in corner $\mathrm{B}$ for 5 of the rats and in corner $\mathrm{C}$ for the remaining 4 rats. The only difference between the control groups was that the control-1 group had one spherical landmark randomly positioned in either corner $\mathrm{B}$ or $\mathrm{C}$, with the stipulations that each corner was used twice during a given session and that the spherical landmark was above the platform for half of the trials in each session. The control-2 group had two identical spherical landmarks, always in corners $\mathrm{B}$ and $\mathrm{C}$. The landmarks were always positioned so that the center of the sphere was situated $25 \mathrm{~cm}$ from the correct corner on a line that bisected the corner (i.e., directly over the 
area where the platform would be placed). The first three trials of Session 14 in Stage 2 were conducted in the same manner as previous trials. The fourth trial of Session 14 was a geometry test. During this test trial, the platform and all landmarks were removed from the pool. The rats were placed in the center of the arena and allowed to swim for $60 \mathrm{sec}$.

Throughout the experiment, except for the test trial, a record was taken of whether, after being released, a rat entered first the correct corner of the pool - the corner containing the platform. A rat was deemed to have entered any of the three corners if its snout crossed a notional circular line with a radius of $40 \mathrm{~cm}$ and with its center at the point where the walls creating the corner met. For ease of exposition, the term correct choice will be used to refer to those occasions when a rat entered the correct corner before any other corner. Also, as an additional measure of performance, latencies to find the platform were recorded. For the purpose of analyzing the results from the test trials, circular search zones were used. Each search zone had a diameter of $30 \mathrm{~cm}$, with its center positioned $25 \mathrm{~cm}$ from a corner on a line that bisected the corner. The percentages of time spent in the correct (i.e., where the platform had been located) and incorrect (i.e., the opposite corner) zones of the triangular-shaped pool were analyzed. A Type I error rate of .05 was adopted for all reported statistical comparisons.

\section{Results and Discussion}

The left-hand panel of Figure 2 shows the mean latencies to find the submerged platform during Stage 1. Rats in each group required less time to find the platform as training progressed. A one-way ANOVA of individual mean latencies for the last three sessions combined revealed a significant difference among the groups $[F(2,24)=14.80]$. Newman-Keuls post hoc tests revealed that the blocking group differed from both the control-1 and control-2 groups, which did not differ. The difference between the blocking and control groups indicates that the platform was more difficult to find when its position was indicated by a spherical rather than a rod-shaped landmark. A possible explanation for this result is that cues that are closer to the goal are more effective for locating it than are those at some distance from the goal (Chamizo \& Rodrigo, 2004).

\section{Stage 1}

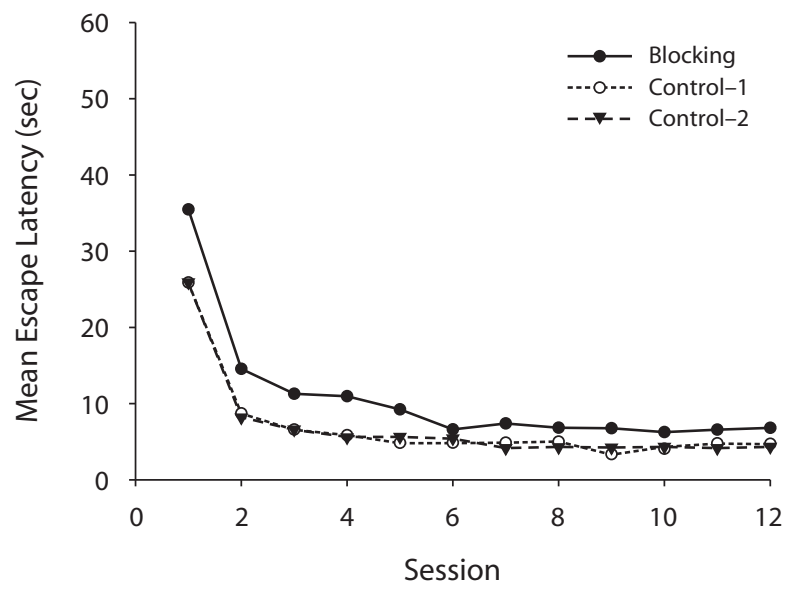

The right-hand panel of Figure 2 shows the latency data from Stage 2. As can be seen, the performance of the blocking group was relatively unaffected by the transition from Stage 1 to Stage 2. The introduction of a new landmark, however, resulted in a disruption of the performance of the two control groups at the outset of Stage 2. A one-way ANOVA based on the individual mean latencies combined across the last three sessions revealed a significant between-group effect $[F(2,24)=4.62]$. NewmanKeuls tests revealed that the blocking group differed from the control-1 group. The two other comparisons were not significant.

The left-hand panel of Figure 3 shows the mean percentages of correct choices for the three groups during the 12 sessions of Stage 1. At first, performance was rather poor, but all three groups rapidly acquired the response of heading to the correct corner after being released into the pool. To compare the performance of the three groups, individual mean percentages of trials on which a correct choice was made were calculated for the last 3 sessions combined. A Kruskal-Wallis test based on these individual means revealed no significant differences among the groups $[H(3)=2.21]$.

The right-hand panel of Figure 3 shows the mean percentages of correct choices for the three groups during the 14 sessions of Stage 2. As can be seen, the blocking group showed excellent transfer of behavior from Stage 1, as compared with the control groups. By the end of training, all groups performed similarly, as confirmed by a Kruskal-Wallis analysis on individual mean percentages for the last 3 sessions combined $[H(3)=3.23]$.

The mean times spent in the correct and incorrect zones for the geometry test are shown in Figure 4. All three groups spent substantially more time in the correct than in the incorrect zone, but the extent of the preference was somewhat less pronounced for the blocking than for the control groups. A $3 \times 2$ (group $\times$ zone) ANOVA revealed a significant main effect of zone $[F(1,24)=57.72]$. The

\section{Stage 2}

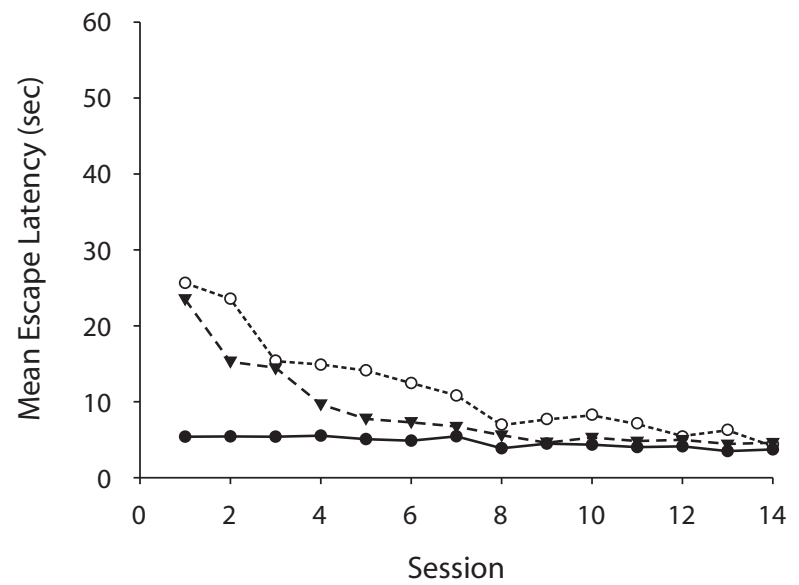

Figure 2. Group mean escape latencies, in seconds, during Stage 1 (left-hand panel) and Stage 2 (right-hand panel) for the blocking, control-1, and control-2 groups of Experiment 1. 

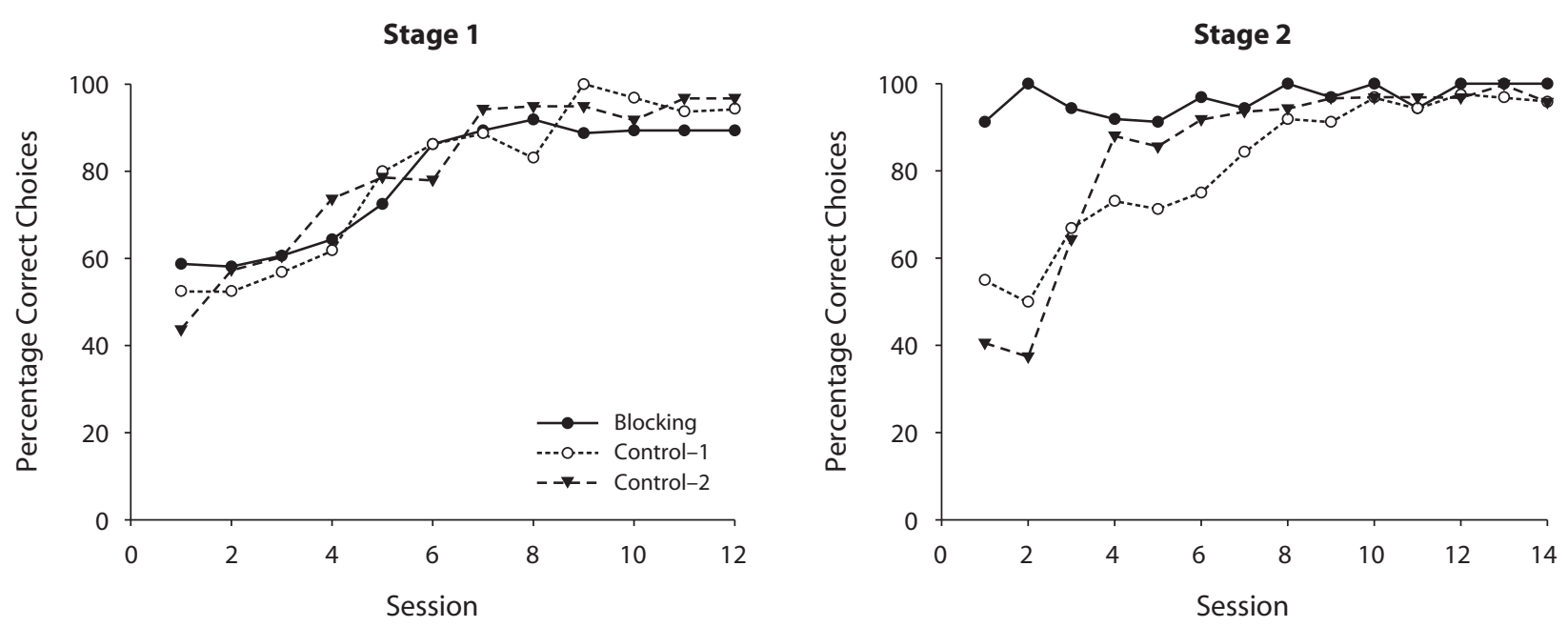

Figure 3. The mean percentages of correct choices made during the training sessions during Stage 1 (left-hand panel) and Stage 2 (right-hand panel) for the blocking, control-1, and control-2 groups of Experiment 1.

remaining analysis revealed a nonsignificant effect for group $[F(2,24)=1.38]$ and a nonsignificant group $\times$ zone interaction $[F(2,24)=1.39]$.

The purpose of this experiment was to test whether the failure of a landmark to block learning about geometric cues was the result of a generalization decrement due to a change in the context between Stages 1 and 2, which reduced the associative strength of the blocking cue. Stages 1 and 2 of the present experiment were conducted in the same context, and still there was no evidence of blocking. This result indicates that a generalization decrement resulting from changes in context between Stages 1 and 2 was not responsible for the failure to observe blocking in the studies of Hayward et al. (2003; Hayward et al., 2004), Pearce et al. (2001), and Wall et al. (2004). An alternative explanation for the failure to reveal blocking in these studies, and the present one, is that insufficient training was given in Stage 1 for the associative strength

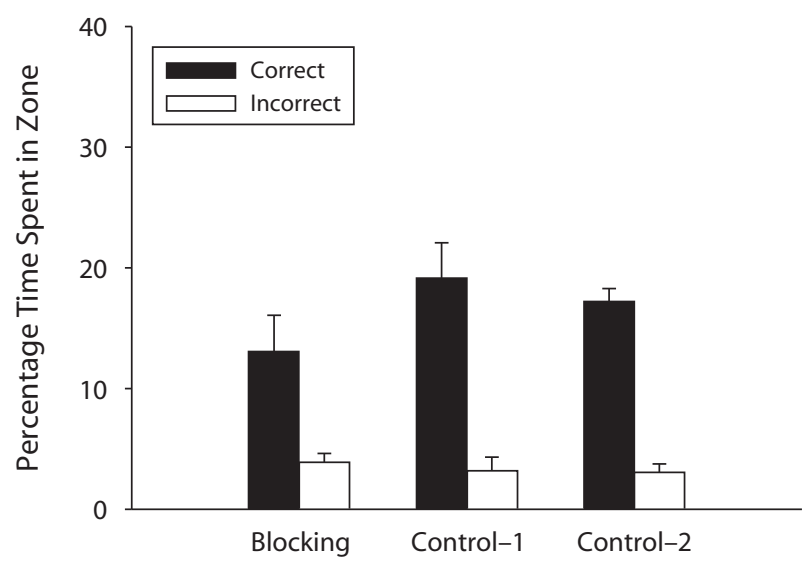

Figure 4. The mean $(+S E M)$ percentages of time spent in the correct and incorrect zones during the geometry test for the three groups of Experiment 1. of the blocking cue to reach asymptote. An obvious way to test this explanation would be to extend the length of training with the blocking cue in Stage 1; this idea served as the rationale for both Experiments 2 and 3.

\section{EXPERIMENT 2}

Experiment 2 was similar to Experiment 1, except that the number of Stage 1 sessions was increased to 24 , in the hope that the additional training would enhance the associative strength of the landmark to such an extent that it would block the geometric cues.

\section{Method}

Subjects and Apparatus. The 16 rats in this experiment were from the same stock and of approximately the same weight as those in Experiment 1, and were housed in the same manner. All of the rats had previously been used in an appetitive conditioning experiment and had no previous training in the swimming pool. At the start of the experiment, they were assigned randomly in equal numbers to two groups. The apparatus was the same as in Experiment 1.

Procedure. The control group was trained in a manner identical to the training of the control-2 group in Experiment 1, and the blocking group was trained in the same manner as its namesake in the previous experiment. The only difference between the experiments was that 24 , rather than 12 , sessions were presented in Stage 1. Stage 2 still consisted of 14 sessions of training. The fourth trial of Session 14 of Stage 2 was a geometry test conducted in the same manner as in Experiment 1.

\section{Results and Discussion}

The left-hand panel of Figure 5 shows the mean escape latencies for the two groups during the 24 sessions of Stage 1. As in Experiment 1, both groups took less time to locate the platform as training progressed. By the end of training, there were no differences between the groups, which was confirmed by a $t$ test using individual mean latencies combined across the last 3 sessions of Stage 1 $[t(14)=1.52]$. The right-hand panel of Figure 5 shows the latencies for Stage 2. An analysis equivalent to that 
Stage 1

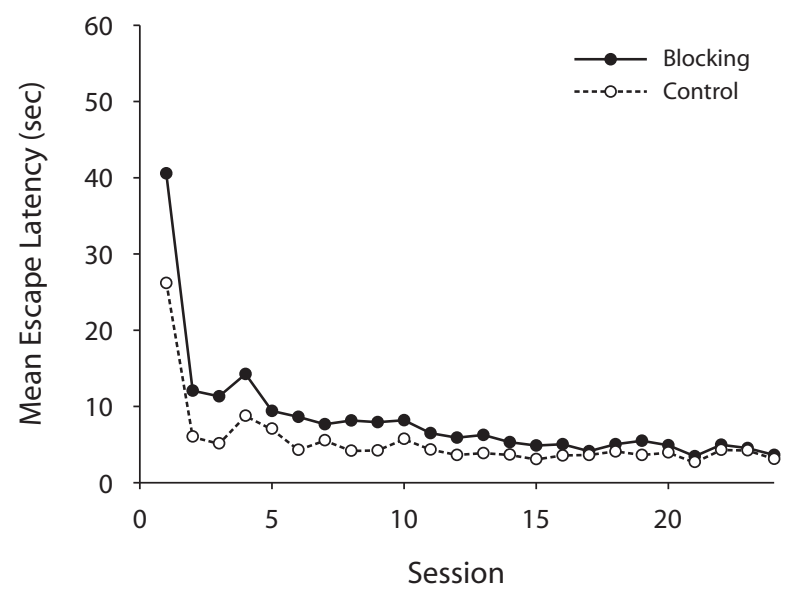

Stage 2

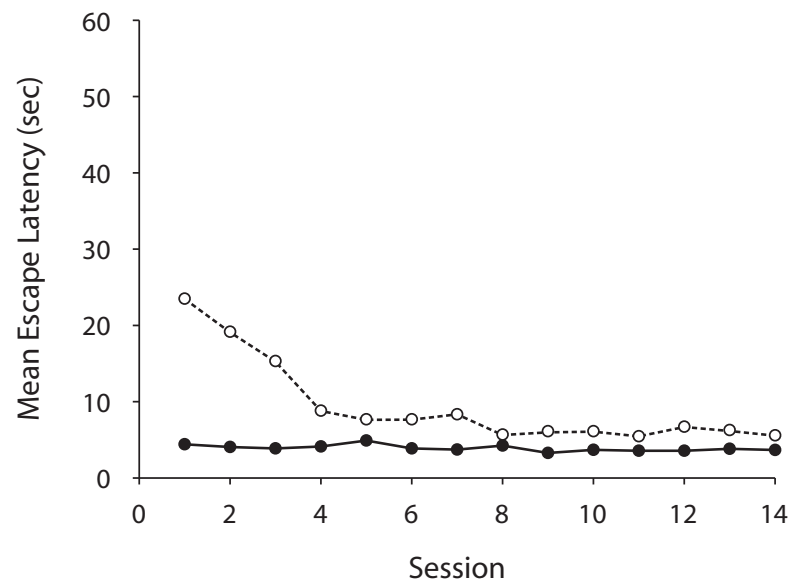

Figure 5. Group mean escape latencies, in seconds, during Stage 1 (left-hand panel) and Stage 2 (right-hand panel) for the blocking and control groups of Experiment 2.

conducted for Stage 1 revealed no significant difference between the groups during the final 3 sessions of this stage $[t(14)=2.03]$.

The left-hand panel of Figure 6 shows the mean percentages of correct choices for the two groups during the 24 sessions of Stage 1. As was seen in Experiment 1, both groups started poorly but eventually acquired the response of heading directly to the correct corner on virtually every trial. To compare the performance of the two groups, individual mean percentages of trials on which a correct choice was made were calculated for the last 3 sessions combined. A Mann-Whitney $U$ test based on these individual means revealed no significant difference between the groups $[U(8,8)=21.50]$.

The right-hand panel of Figure 6 shows the mean percentages of correct choices for the two groups during the 14 sessions of Stage 2. As in Experiment 1, the perfor- mance of the control group was impaired at the beginning of Stage 2 relative to the blocking group. To compare the performance of the two groups, individual mean percentages of trials on which a correct choice was made were calculated for the last 3 sessions combined, revealing no significant difference [Mann-Whitney $U(8,8)=$ 23.00].

Figure 7 shows the results from the geometry test. The blocking group spent a smaller proportion of time in the correct zone than did the control group. A $2 \times 2$ (group $\times$ zone) ANOVA revealed significant effects of group $[F(1,14)=8.57]$ and zone $[F(1,14)=60.27]$ and a significant group $\times$ zone interaction $[F(1,14)=8.37]$. A simple-effects analysis of the interaction revealed that the blocking group spent significantly less time in the correct zone than did the control group $[F(1,28)=16.53]$. Both groups also spent significantly more time searching
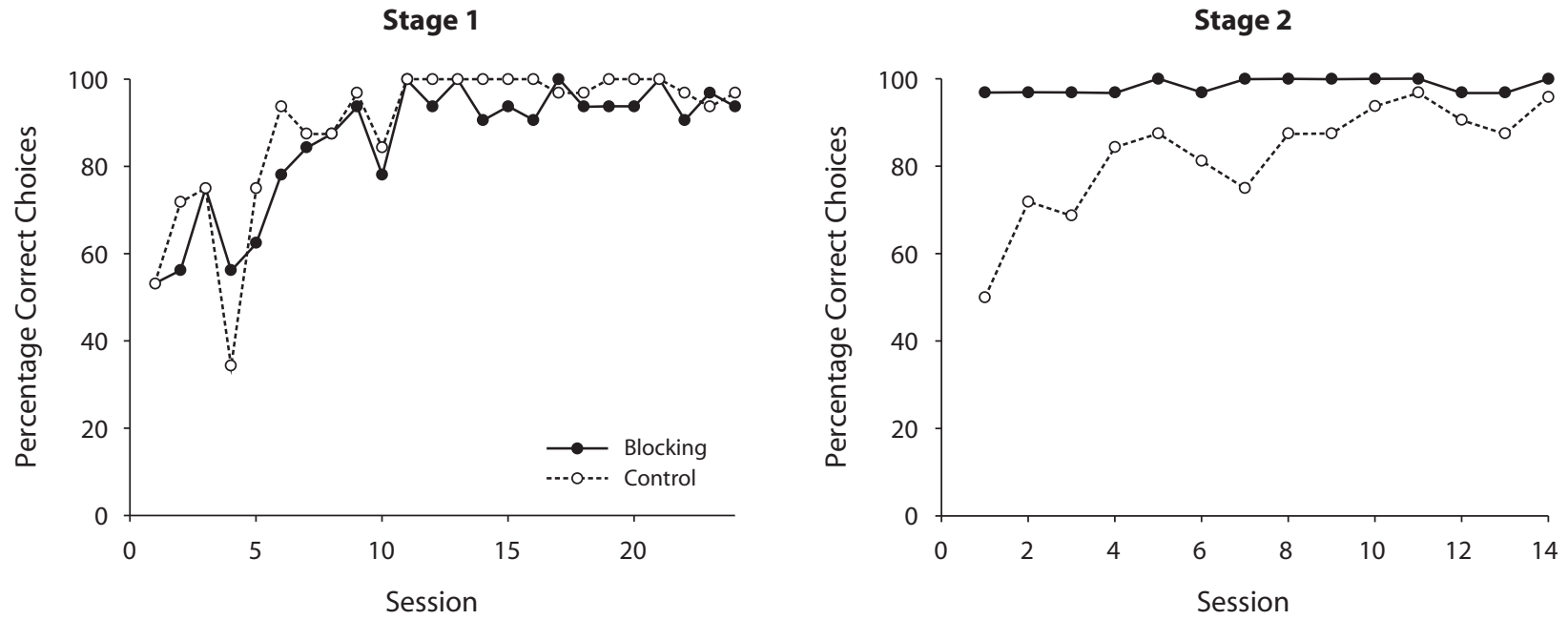

Figure 6. The mean percentages of correct choices made during the training sessions during Stage 1 (left-hand panel) and Stage 2 (right-hand panel) for the blocking and control groups of Experiment 2. 


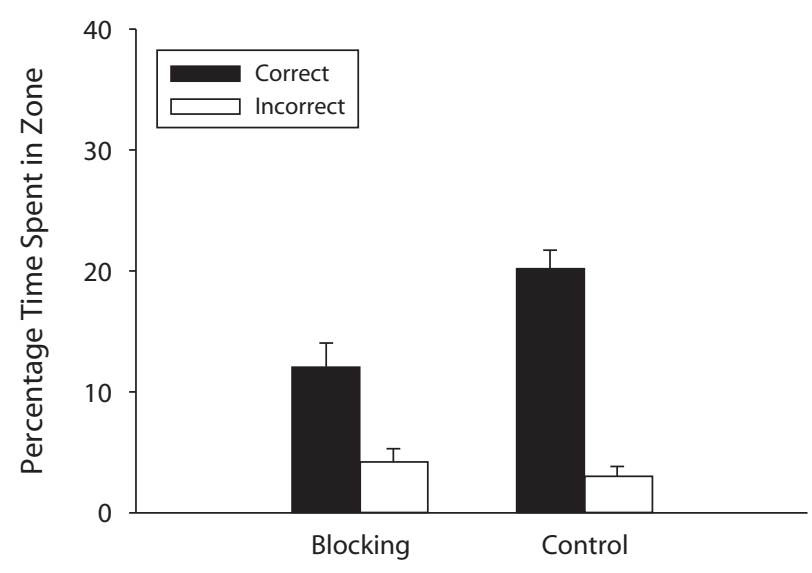

Figure 7. The mean $(+S E M)$ percentages of time spent in the correct and incorrect zones during the geometry test for the two groups of Experiment 2.

for the platform in the correct than in the incorrect zone $[F \mathrm{~S}(1,14)>11.86]$.

Extended Stage 1 training with the landmark resulted in the blocking group spending significantly less time than the control group in the vicinity of the correct corner during the test trial. The results demonstrate that with 24 sessions of training with the landmark during Stage 1 of a blocking experiment, the landmark can block learning about geometric cues during the second stage of the experiment. This finding constitutes the first demonstration with animals that the presence of a landmark, given suitable training, can block learning about geometric cues (for a similar demonstration of blocking with humans, see Wilson \& Alexander, 2008). It now becomes important to consider how such blocking took place. According to the model of Miller and Shettleworth (2007), the extensive pretraining with the landmark endowed it with sufficient associative strength to enable it to restrict learning about the geometric cues, because both sets of cues were in competition for a limited amount of associative strength (Rescorla \& Wagner, 1972). It is possible that the extended training did in fact increase the associative strength of the landmark. Behavioral evidence of this comes from the latencies to find the platform during Stage 1. In Experiment 1 , when rats were given only 12 sessions of training with the landmark during Stage 1, the latencies to find the platform were significantly longer for the blocking group than for the control group. In the present study, after initial training of 24 sessions with the landmark, no differences in latencies were observed between the groups. Moreover, in Experiment 2, an analysis of the latencies for Sessions 10,11 , and 12 combined revealed a difference between the blocking and control groups similar to the one in Experiment $1[t(14)=3.55]$. Thus, the extended training was effective at reducing the latencies to find the platform, which may reflect an increase in the associative strength of the landmark with extended training.

However, it is possible to explain the present results in a rather different way. Note that training in Stages 1 and 2 took place in the same triangular environment. According to the theory of Mackintosh (1975), the protracted Stage 1 training would result in attention to the geometric cues being low because they were irrelevant for finding the platform (Mackintosh, 1973). On the other hand, attention to the landmark used in Stage 1 would be high because it was a reliable cue for finding the platform. Upon the introduction of Stage 2, the experimental group, according to this theory, would continue to pay attention to the landmark and ignore the geometric cues and, despite their now being relevant, the geometric cues should gain little associative strength. In contrast, the introduction of a new landmark at the outset of Stage 2 for the control group would result in neither the landmark nor the geometric cues being good predictors of where the platform could be found. As a consequence, this theory predicts that attention to the geometric cues would increase in the control group and permit them to gain more associative strength than in the experimental group.

Support for this account can be found in reports by Prados, Redhead, and Pearce (1999) and Redhead, Prados, and Pearce (2001). Redhead et al., for example, trained two groups of rats to swim to a submerged platform beneath a landmark in a circular pool while they could view the stimuli provided by the room housing the pool. For one group, the platform remained in the same place for the four trials of each session, whereas for another group the platform was moved from trial to trial. The room cues were thus relevant for locating the platform within a session for the first but not for the second group. During a subsequent test with the platform in a fixed location for both groups, but without the landmarks, the first group showed a more rapid decline in escape latencies across trials than did the second group. Redhead et al. attributed this outcome to the different treatments resulting in the first group paying more attention to the room cues at the outset of the test trials than did the second group.

\section{EXPERIMENT 3}

The results from Experiment 2 demonstrate blocking, but it is not clear whether this effect was a consequence of stimuli competing for associative strength or of changes in attention to the geometric cues. As a step toward evaluating these explanations, the present experiment was based on the design of Experiment 2, except that the training in Stage 1 was conducted in a circular rather than a triangular pool. Provided that the training in Stage 1 endows the landmark with sufficient associative strength, the model of Miller and Shettleworth (2007) predicts that blocking should be observed after training in either environment. On the other hand, in the present experiment, the novelty of the geometric cues at the outset of Stage 2 can be expected to encourage the blocking group to pay them considerable attention, and according to the attentional explanation that has just been developed, the likelihood of observing blocking should here be reduced.

A comparison of the results of Experiments 1 and 2 implies that the landmark blocked learning about the geometric cues when there were 24 , but not when there were only 12 , sessions of training in Stage 1 . To confirm this 
conclusion, the present experiment included groups that received 12 and 24 sessions of training in Stage 1. The experiment therefore contained four groups, all of which were initially trained in a circular swimming pool during Stage 1. The two blocking groups received a spherical landmark directly above the platform, randomly positioned across trials in one of the four quadrants of the pool. One of these groups received 12 sessions of training, and the other received 24 sessions. The two control groups received the same training, but rather than the spherical landmark, they received a rod-shaped landmark attached to the platform. Stage 2 was conducted in the same way as for the blocking and control groups of Experiment 2.

In addition to the geometry test, landmark tests were conducted to give an indication of the associative strength acquired by the spherical landmark for each of the groups. The blocking groups are of particular interest, because the associative strength of the landmark should differ according to the amount of training given in Stage 2 if blocking were to be found in the group that received more Stage 1 training.

\section{Method}

Subjects and Apparatus. The 32 rats in this experiment were from the same stock and of approximately the same weight as those in Experiment 1, and were housed in the same manner. All rats had previously been used in an appetitive conditioning experiment and had no previous training in the swimming pool. At the start of the experiment, they were assigned randomly in equal numbers to four groups. The apparatus was the same as in Experiment 1.

Procedure. All procedures were identical to those of Experiment 2, with the exceptions that Stage 1 was conducted in a circular rather than a triangular pool and that half of the rats received 12 sessions of Stage 1 training (groups control-12 and blocking-12) and the other half received 24 (groups control-24 and blocking-24). The groups that received 24 sessions of Stage 1 training were started 12 days prior to those that received 12 sessions, to ensure that Stage 2 commenced on the same day for all rats. During Stage 1, the control groups received the rod attached to the platform, which was placed randomly in the different quadrants of the circular pool, with only the stipulation that each quadrant was used once within a session. The platform was positioned 25 or $50 \mathrm{~cm}$ from the side of the pool, each for two trials per session. The blocking groups received identical treatment, except that the landmark was a sphere suspended directly over the platform. Stage 2 was conducted in the same manner as in Experiment 2 and contained 16 sessions. The last trial of Session 14 of Stage 2 was a geometry test, conducted in the same manner as in Experiment 1. The first three trials of Session 16 were conducted in the same manner as the previous trials, but the fourth trial was a landmark test. For this test trial, the platform and the walls forming the triangular-shaped pool were removed. The spherical landmark was hung $50 \mathrm{~cm}$ from the edge of the circular pool. A circular search zone $(30 \mathrm{~cm}$ in diameter) directly under the landmark was used to analyze the results from this test. Session 16 was then followed by three additional tests with the landmark, at the rate of one per day.

\section{Results and Discussion}

The left-hand panel of Figure 8 shows the mean escape latencies for the four groups during the 24 sessions of Stage 1. In contrast to the previous experiments, we did not record choices during Stage 1, because it took place in a circular pool. All groups learned rapidly to swim to the platform by the end of the stage. A $2 \times 2$ (group $\times$ session) ANOVA conducted on the individual mean escape latencies combined across the last 3 sessions revealed significant effects of group $[F(1,28)=48.59]$ and session $[F(1,28)=7.15]$, as well as a significant group $\times$ session interaction $[F(1,28)=6.07]$. An analysis of the interaction revealed that the blocking-12 group took significantly longer to find the platform than did the blocking-24 group $[F(1,28)=13.20]$, but the control-12 and control-24 groups did not differ $(F<1)$. Given that the blocking-24 group found the platform faster than the blocking-12 group, it could be inferred that extended training was effective in the blocking-24 group, resulting in the associative strength of the sphere being greater in this group than in the blocking-12 group. The righthand panel of Figure 8 shows the latencies for Stage 2. An analysis equivalent to that of Stage 1 on the last 3 sessions
Stage 1

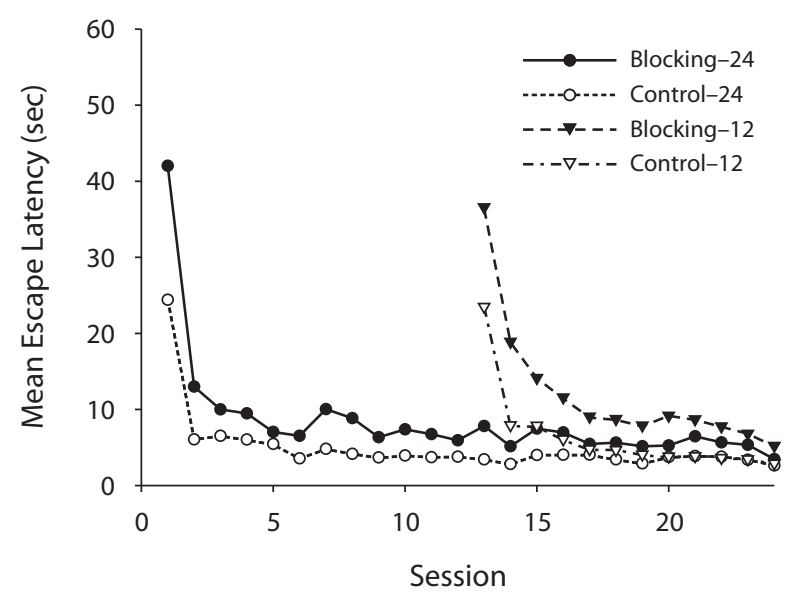

Stage 2

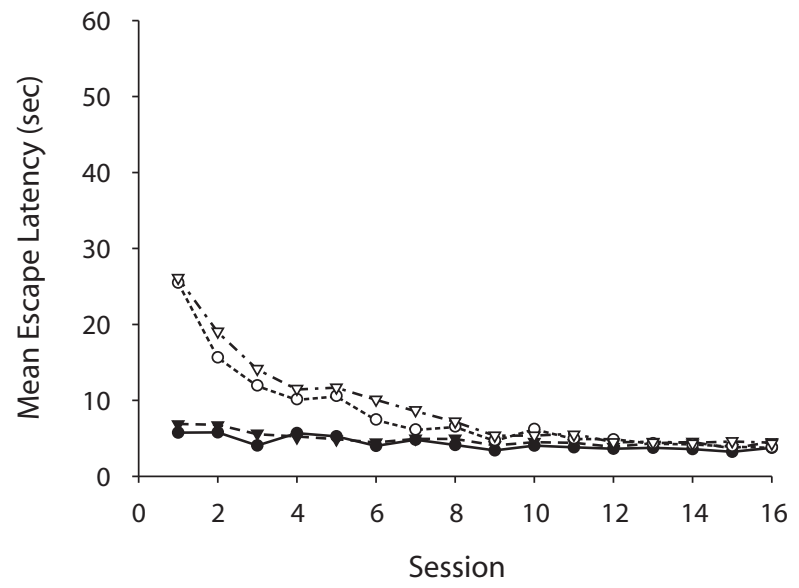

Figure 8. Group mean escape latencies, in seconds, during Stage 1 (left-hand panel) and Stage 2 (right-hand panel) for the four groups of Experiment 3. 


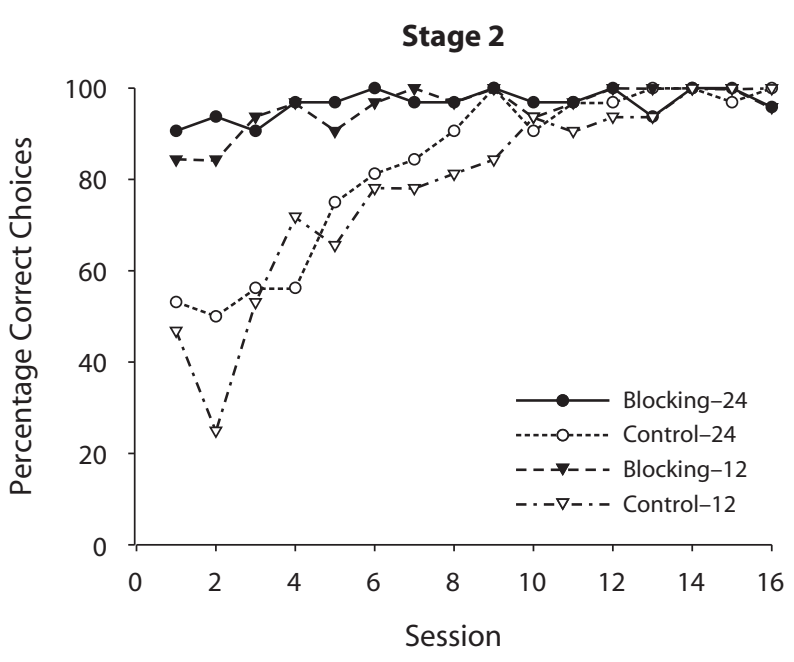

Figure 9. The mean percentages of correct choices made during the training sessions during Stage 2 for the four groups of Experiment 3.

before the first test trial revealed no significant effect of group $[F(1,28)=3.45]$ or session $[F(1,28)=1.59]$, as well as a nonsignificant group $\times$ session interaction $[F(1,28)=1.92]$.

Figure 9 shows the mean percentages of correct choices for the four groups during the 16 sessions of Stage 2. At first, the control groups made a correct choice on approximately half of the trials, whereas the blocking groups made a correct choice on the majority of trials from the outset of Stage 2. A Kruskal-Wallis test based on the individual means of correct choices, combined across the last 3 sessions before the first test trial, revealed no significant differences among the groups $[H(4)=6.55]$.

\section{Geometry Test}

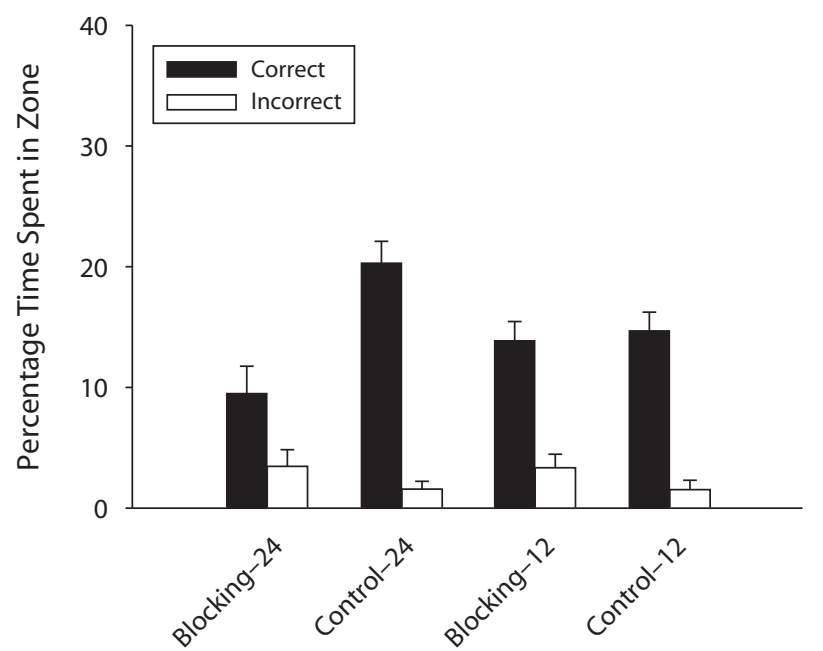

The left-hand panel of Figure 10 shows the times spent searching in the correct and incorrect zones for all four groups during the geometry test. It is obvious that the blocking-24 group spent less time in the correct search zone than did its respective control, whereas the blocking12 and control-12 groups spent equal amounts of time in the correct zone. A $2 \times 2 \times 2$ (group $\times$ session $\times$ zone) ANOVA was conducted (in which group refers to blocking or control; session refers to 12 or 24 sessions during Stage 1; and zone refers to correct or incorrect zones). The analysis revealed significant effects of group $[F(1,28)=$ $4.57]$ and zone $[F(1,28)=121.67]$. There were also significant two-way interactions of group $\times$ session $[F(1,28)=$ $7.51]$ and group $\times$ zone $[F(1,28)=12.41]$. However, more importantly, there was a significant group $\times$ session $\times$ zone three-way interaction $[F(1,28)=5.25]$. The remaining findings from the ANOVA were nonsignificant $\left(F_{\mathrm{S}}<1\right)$.

A simple-effects analysis of the three-way interaction revealed that groups control-24 and blocking-24 differed in the percentages of time spent in the correct zone $[F(1,56)=28.80]$. This difference was not present in groups that received only 12 sessions of training in Stage 1 $(F<1)$. Also, the blocking-24 group spent significantly less time in the correct zone than did the blocking-12 group $[F(1,56)=4.91]$. Unexpectedly, the control-12 group spent significantly less time in the correct zone than did the control-24 group $[F(1,56)=7.57]$.

The right-hand panel of Figure 10 shows times spent in the correct zone during the landmark test. The blocking groups spent larger proportions of time searching directly under the landmark than did the control groups. A $2 \times 2$ ANOVA (group $\times$ session) revealed a significant group effect $[F(1,28)=33.87]$. The session effect and the group $\times$ session interaction were nonsignificant $(F \mathrm{~s}<1)$.

Figure 10. The mean $(+S E M)$ percentages of time spent in the correct and incorrect zones during the geometry test of Experiment 3 (left-hand panel) and the mean $(+S E M)$ percentages of time spent in the correct zone during the landmark test of Experiment 3 (right-hand panel). 


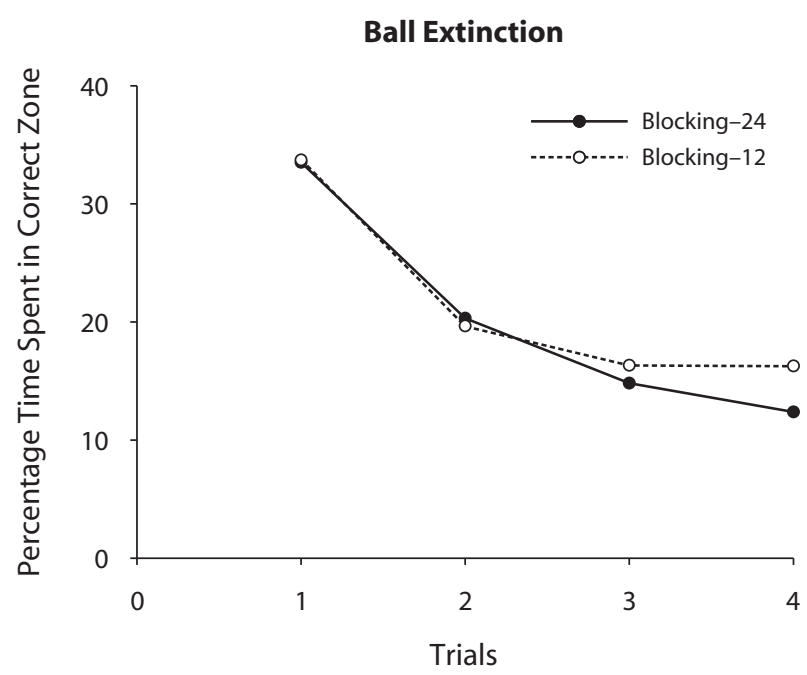

Figure 11. The mean percentages of time spent in the correct zone for the four extinction trials for the landmark among the blocking-24 and blocking-12 groups of Experiment 1.

Figure 11 shows the times spent searching in the correct zone for the blocking-24 and blocking-12 groups across the four consecutive landmark tests. As can be seen, both groups spent less time searching under the landmark with each succeeding trial. A $2 \times 2$ (group $\times$ trial) ANOVA confirmed these results: There was a significant effect of trial $[F(3,14)=29.81]$, but the group effect and the group $\times$ trial interaction were nonsignificant $(F \mathrm{~s}<1)$.

We successfully replicated the results obtained in Experiments 1 and 2. Rats that received 24 sessions of training with the spherical landmark during Stage 1 spent significantly more time in the correct corner during the test in the triangle than did the control groups. This difference was not evident between the two groups trained for 12 sessions in Stage 1. These results suggest that the blocking effect observed in Experiment 2 did not depend on a loss of associability by the geometric cues by virtue of their being present during Stage 1. According to this explanation, blocking in Experiment 2 occurred because the training in Stage 1 made the blocking group fail to attend to the geometric cues in Stage 2. By introducing the triangular arena at the outset of Stage 2, we anticipated that its geometric features would be attended to by both groups, at least initially. These features would then be expected to acquire associative strength, making it difficult to reveal blocking in the blocking-24 group.

One unexpected finding from the experiment was that the control group that received 12 sessions of training in Stage 1 spent less time in the correct search zone during the test trial in the triangle than did the control group that received 24 sessions. Both groups received the same amount of identical training during Stage 2 in the triangle, and thus there is no good reason to expect them to differ in the degree to which the geometric cues provided by the triangle controlled searching for the platform. It is difficult to think of a good explanation for this difference between the results of the two control groups, except that it might be due to sampling error. Nonetheless, this unexpected finding does not affect the principal conclusions we draw from this experiment. The significantly less time spent in the correct zone by the blocking-24 than by the control-24 group during the first test demonstrates that blocking was effective after 24 sessions of training in Stage 1. Furthermore, the difference between the results from the first test trial for the blocking-24 and blocking-12 groups demonstrates that blocking was more effective after 24 sessions of Stage 1 training than after 12 sessions. A second unexpected result from the experiment was the failure to find a difference between the two blocking groups during the tests with the landmark in the circular pool. The implications of this finding will be considered shortly.

\section{GENERAL DISCUSSION}

The present set of experiments has provided the first demonstration of blocking of geometric cues by a discrete landmark in animals. We demonstrated in two experiments that if rats are first trained to locate a submerged platform underneath a landmark for 24 sessions, learning about the position of the platform with reference to the shape of the environment is blocked in a second stage when both the shape of the environment and the landmark indicate where the platform can be found. Before considering the theoretical implications of these results, some discussion is needed as to whether the experiments actually revealed blocking.

The control groups in Stage 2 of Experiments 2 and 3 were trained with a spherical landmark in each of the corners at the base of a triangular pool, with the platform located beneath one of them. This treatment was different from the conventional treatment for a control group in a blocking design, which would involve the same Stage 2 treatment given to the blocking group, to control for overshadowing. We intentionally used an unconventional control condition because a failure to find a difference between the test results of the blocking and the control groups in these novel conditions would reveal particularly forcefully that the blocking treatment did not restrict learning based on the geometric cues. As it turns out, there was a difference between the test results for the blocking and control groups in Experiments 2 and 3, and given the design of the control condition, it is impossible to claim with certainty that this difference was a consequence of blocking rather than overshadowing. That is, the pretraining with the landmark in Stage 1 may not have been responsible for the poor control gained by geometric cues over the search for the platform in Stage 2. Instead, this outcome may have occurred because the single landmark overshadowed the geometric cues during Stage 2 for the blocking group. Such overshadowing would not be anticipated for the control groups, since the manners in which the landmarks were used meant that subjects in those groups had to rely on the shape of the pool for finding the platform. There are two reasons, however, for believing that the difference between the blocking and control groups of Experiments 2 and 3 was not a consequence of overshadowing. On the one hand, there is no obvious 
reason why overshadowing in Stage 2 should be more effective after 24 than after 12 sessions of Stage 1 training, yet this would have to be the case for the absence of blocking in Experiment 1 to be explained. On the other hand, McGregor, Horne, Esber, and Pearce (in press) trained two groups of rats identically to the blocking and control groups of our Experiments 2 and 3, except that Stage 1 training was omitted, and they found no hint of overshadowing. It seems, therefore, that the difference between the effects of the control and blocking treatments in the present experiments depended on the extended training in Stage 1 with the spherical landmark, which implies that the outcome of Experiments 2 and 3 was a consequence of blocking rather than overshadowing.

We noted in the introduction that blocking of geometric cues has been reported using the color of the walls of a distinctively shaped arena as the additional cue for finding a hidden goal (Pearce et al., 2006). In contrast to the present experiments, this effect was reported with only 14 sessions of Stage 1 training. A possible explanation for the present finding that 24 , but not 12 , sessions of Stage 1 training were necessary to obtain blocking is that the salience of the (relatively small) spherical landmark was less than that of the (relatively large) black and white walls used in Pearce et al.'s study. More initial training would then be required with the sphere than with the black and white walls, in order to endow the sphere with sufficient associative strength for it to block the geometric cues.

Our experiments were conducted to test the suggestion by Miller and Shettleworth (2007) that previous failures of nongeometric cues to block geometric cues were a consequence of the former cues possessing inadequate associative strength at the start of Stage 2. Miller and Shettleworth further proposed that if the blocking cue should gain sufficient associative strength during Stage 1, it would block spatial learning about the geometric cues for reasons that follow from the Rescorla-Wagner (1972) theory. The fact that blocking was effective after 24 but not 12 sessions of Stage 1 training is consistent with this claim, but as noted earlier, not all of our findings are compatible with this explanation. For example, the test in the triangle at the end of Experiment 3 revealed that the blocking-24 group spent less time in the correct corner than did the blocking-12 group. The implication of this finding is that the associative strength of the spherical landmark at the time of testing was greater in the blocking-24 than in the blocking-12 group. However, a repeated series of test trials with the spherical landmark indicated that its associative properties were very similar in both groups. A possible explanation for this outcome is that the test for the associative properties of the sphere was insensitive. Although it is not possible to refute this explanation, its force is muted by the outcome of the tests for the two control groups. During Stage 2, the sphere was a less reliable cue for finding the platform in the two control groups than in the two blocking groups, and the associative strength of the sphere should therefore have been weaker in the two controls than the blocking groups. The results shown in Figure 10 confirm this prediction, which demonstrates that the test in the circular pool was at least sufficiently sensitive to detect some between-group differences in the associative strength of the sphere.

As an alternative to a competition between the sphere and the geometric cues for associative strength, it is possible that the present results were a consequence of these cues competing for attention instead. Thus, it is conceivable that rats in the blocking groups paid considerably more attention to the sphere after 24 than after 12 sessions of Stage 1 training. Blocking rats that received only 12 sessions of pretraining would then have paid more attention to the geometric cues at the start of Stage 2 than did those receiving 24 sessions of pretraining, and thus would have learned more about the position of the platform with reference to these cues. A benefit of this explanation is that it does not necessarily predict a difference in the associative strengths acquired by the landmark in the blocking-12 and blocking-24 groups. It is quite possible that the training in Stage 2 allowed conditioning in both groups with this cue to reach the same asymptote. A further benefit of this attentional explanation is that it does not necessarily predict that the presence of the sphere will overshadow learning about the significance of geometric cues for finding the platform. If extended training is required with the sphere by itself before it distracts attention away from the shape of the environment, it would then follow that overshadowing between these two cues would not be seen if they were presented in compound from the outset of training. It was noted above that McGregor et al. (in press) failed to find any evidence that the spherical landmark overshadowed spatial learning based on the geometric cues provided by a triangular arena.

On the basis of appetitive conditioning experiments conducted in a rectangular arena, Cheng (1986; see also Gallistel, 1990) argued that spatial learning based on the shape of the environment progresses independently of learning about nongeometric cues (but see Cheng, 2008, for a retraction of this view). More recently, a similar point of view has been expressed by Doeller, King, and Burgess (2008). Basing their argument on findings from the performance of humans in a virtual maze, these researchers suggested that learning about the significance of boundaries for finding a hidden goal will be unaffected by the presence of nonboundary cues that also signal where the goal is located. An implication of both sets of proposals is that blocking should not have been observed in the present experiments and, at first sight, our finding to the contrary appears to cast doubt on them. However, if the blocking effects we have reported were a consequence of attentional processes rather than of an error-correcting learning algorithm (see, e.g., Rescorla \& Wagner, 1972), it may be possible to reconcile our results with the point of view that something is special about spatial learning based on shapes or boundaries. Perhaps learning about the position of a hidden goal with reference to these cues does not compete with learning based on other cues, and effects such as blocking and overshadowing, when they occur, are a consequence of animals' failure to pay heed to these cues because attention is directed elsewhere. As they stand, the present experiments do not allow us to reject this possibility. 
The results from our experiments lend support to the assertion by Miller and Shettleworth (2007) that previous failures to observe blocking of geometric cues by discrete landmarks were a consequence of inadequate Stage 1 training with the landmark. There is rather less support from the results, however, for the additional assertion of Miller and Shettleworth that blocking of geometric cues, when it occurs, is due to a competition between the cues for a limited pool of associative strength (see, e.g., Rescorla \& Wagner, 1972). Given the possibility that attentional processes might also have been responsible for the effects we have reported, it might be a mistake to look to blocking as a tool for assessing whether learning about geometric cues provided by the shape of the environment is governed by a rule such as that advocated by Rescorla and Wagner. Instead, alternative tests should be sought whose outcome, if successful, may not be so readily explained in terms of attentional processes.

\section{AUTHOR NOTE}

This research was supported by a grant from the United Kingdom Medical Research Council and by a United Kingdom Overseas Research Studentship. Correspondence concerning this article should be addressed to either M. R. Horne or J. M. Pearce, School of Psychology, Cardiff University, Cardiff CF10 3YG, Wales (e-mail: hornemr@cardiff .ac.uk or pearcejm@cardiff.ac.uk).

\section{REFERENCES}

Chamizo, V. D., \& Rodrigo, T. (2004). Effect of absolute spatial proximity between a landmark and a goal. Learning \& Motivation, 35, $102-114$.

Chamizo, V. D., Sterio, D., \& Mackintosh, N. J. (1985). Blocking and overshadowing between intra-maze and extra-maze cues: A test of the independence of locale and guidance learning. Quarterly Journal of Experimental Psychology, 37B, 235-253.

CHENG, K. (1986). A purely geometric module in the rat's spatial representation. Cognition, 23, 149-178.

Cheng, K. (2008). Whither geometry? Troubles of the geometric module. Trends in Cognitive Sciences, 12, 355-361.

Cheng, K., \& Newcombe, N. S. (2005). Is there a geometric module for spatial orientation? Squaring theory and evidence. Psychonomic Bulletin \& Review, 12, 1-23.

Doeller, C. F., King, J. A., \& Burgess, N. (2008). Parallel striatal and hippocampal systems for landmarks and boundaries in spatial memory. Proceedings of the National Academy of Sciences, 105 , 5915-5920.

Gallistel, C. R. (1990). The organization of learning. Cambridge, MA: MIT Press.

Hayward, A., Good, M. A., \& Pearce, J. M. (2004). Failure of a landmark to restrict spatial learning based on the shape of the environment. Quarterly Journal of Experimental Psychology, 57B, 289-314.

Hayward, A., McGregor, A., Good, M. A., \& Pearce, J. M. (2003). Absence of overshadowing and blocking between landmarks and the geometric cues provided by the shape of a test arena. Quarterly Journal of Experimental Psychology, 56B, 114-126.

Kamin, L. J. (1969a). Predictability, surprise, attention, and conditioning. In B. A. Campbell \& R. M. Church (Eds.), Punishment and aversive behavior (pp. 279-296). New York: Appleton-Century-Crofts.

Kamin, L. J. (1969b). Selective association and conditioning. In N. J. Mackintosh \& W. K. Honig (Eds.), Fundamental issues in associative learning: Proceedings of a symposium held at Dalhousie University,
Halifax, June 1968 (pp. 42-64). Halifax, NS: Dalhousie University Press.

Larkin, M. J. W., Aitken, M. R. F., \& Dickinson, A. (1998). Retrospective revaluation of causal judgments under positive and negative contingencies. Journal of Experimental Psychology: Learning, Memory, \& Cognition, 24, 1331-1352.

Mackintosh, N. J. (1973). Stimulus selection: Learning to ignore stimuli that predict no change in reinforcement. In R. A. Hinde \& J. Stevenson-Hinde (Eds.), Constraints on learning: Limitations and predispositions (pp. 75-96). London: Academic Press.

Mackintosh, N. J. (1975). A theory of attention: Variations in the associability of stimuli with reinforcement. Psychological Review, 82, 276-298.

Mackintosh, N. J., \& Honig, W. K. (1970). Blocking and enhancement of stimulus control in pigeons. Journal of Comparative \& Physiological Psychology, 73, 78-85.

McGregor, A., Horne, M. R., Esber, G. R., \& Pearce, J. M. (in press). Absence of overshadowing between a landmark and geometric cues in a distinctively shaped environment. Journal of Experimental Psychology: Animal Behavior Processes.

Miller, N. Y., \& Shettleworth, S. J. (2007). Learning about environmental geometry: An associative model. Journal of Experimental Psychology: Animal Behavior Processes, 33, 191-212.

MorRis, R. G. M., \& Spooner, R. I. W. (1990). WaterMaze [Computer software]. Edinburgh: WaterMaze Software.

Pearce, J. M., Graham, M., Good, M. A., Jones, P. M., \& McGregor, A. (2006). Potentiation, overshadowing, and blocking of spatial learning based on the shape of the environment. Journal of Experimental Psychology: Animal Behavior Processes, 32, 201-214.

Pearce, J. M., Ward-Robinson, J., Good, M., Fussell, C., \& Aydin, A. (2001). Influence of a beacon on spatial learning based on the shape of the test environment. Journal of Experimental Psychology: Animal Behavior Processes, 27, 329-344.

Prados, J., Redhead, E. S., \& Pearce, J. M. (1999). Active preexposure enhances attention to the landmarks surrounding a Morris swimming pool. Journal of Experimental Psychology: Animal Behavior Processes, 25, 451-460.

Redhead, E. S., Prados, J., \& Pearce, J. M. (2001). The effects of pre-exposure on escape from a Morris pool. Quarterly Journal of Experimental Psychology, 54B, 353-367.

Rescorla, R. A., \& Wagner, A. R. (1972). A theory of Pavlovian conditioning: Variations in the effectiveness of reinforcement and nonreinforcement. In A. H. Black \& W. F. Prokasy (Eds.), Classical conditioning II: Current research and theory (pp. 64-99). New York: Appleton-Century-Crofts.

Roberts, A. D. L., \& Pearce, J. M. (1999). Blocking in the Morris swimming pool. Journal of Experimental Psychology: Animal Behavior Processes, 25, 225-235.

Rodrigo, T., Chamizo, V. D., McLaren, I. P. L., \& Mackintosh, N. J. (1997). Blocking in the spatial domain. Journal of Experimental Psychology: Animal Behavior Processes, 23, 110-118.

Tennant, W. A., \& Bitterman, M. E. (1975). Blocking and overshadowing in two species of fish. Journal of Experimental Psychology: Animal Behavior Processes, 1, 22-29.

Wall, P. L., Botly, L. C. P., Black, C. K., \& Shettleworth, S. J. (2004). The geometric module in the rat: Independence of shape and feature learning in a food finding task. Learning \& Behavior, 32, 289-298.

WILLNER, J. A. (1978). Blocking of a taste aversion by prior pairings of exteroceptive stimuli with illness. Learning \& Motivation, 9, 125-140.

Wilson, P. N., \& Alexander, T. (2008). Blocking of spatial learning between enclosure geometry and a local landmark. Journal of Experimental Psychology: Learning, Memory, \& Cognition, 34, 1369-1376.

(Manuscript received July 25, 2008;

revision accepted for publication September 11, 2008.) 